\title{
Implications of the measurement of pulsars with two solar masses for quark matter in compact stars and HIC. A NJL model case study.
}

\author{
T. Klähn, ${ }^{1, *}$ R. Eastowiecki, ${ }^{1,+}$ and D. Blaschke ${ }^{1,2,3,+}$ \\ ${ }^{1}$ Instytut Fizyki Teoretycznej, Uniwersytet Wroctawski, PL-50-204 Wroctaw, Poland \\ ${ }^{2}$ Bogoliubov Laboratory for Theoretical Physics, JINR Dubna, RU-141980 Dubna, Russia \\ ${ }^{3}$ Fakultät für Physik, Universität Bielefeld, D-33615 Bielefeld, Germany
}

(Dated: October 11, 2018)

\begin{abstract}
The precise measurement of the high masses of the pulsars PSR J1614-2230 $\left(M_{1614}=\right.$ $\left.1.97 \pm 0.04 M_{\odot}\right)$ and PSR J0348-0432 $\left(M_{0348}=2.01 \pm 0.04 M_{\odot}\right)$ provides an important constraint for the equation of state of cold, dense matter and is suited to give interesting insights regarding the nature and existence of the possible phase transition to deconfined quark matter in the cores of neutron stars. We analyze the stability and composition of compact star sequences for a class of hybrid nuclear - quark-matter equations of state. The quark matter phase is described in the framework of a standard color superconducting 3-flavor Nambu-Jona-Lasinio model and the hadronic phase is given by the Dirac-BruecknerHartree-Fock equation of state for the Bonn-A potential. The phase transition is obtained by a Maxwell construction. Within this model setup we aim to constrain otherwise not strictly fixed parameters of the NJL model, namely the coupling strengths in the vector meson and diquark interaction channels. We perform this investigation for two different parameterizations characterized by a different scalar coupling constant. The analysis of flow data obtained in heavy-ion collisions resulted in a further constraint which we account for in our discussion. Massive hybrid stars with extended quark matter cores can be obtained in accordance with all of the considered constraints.

PACS numbers: 12.38.Mh,12.38.Lg,26.60.Kp,97.60.Jd,25.75.Ag
\end{abstract}

\section{INTRODUCTION}

Neutron stars (NS) are considered to be cosmic laboratories for dense matter [1, 2]. Of particular interest is the fact that the region of high densities and very low temperatures where NS

*Electronic address: thomas.klaehn@ift.uni.wroc.pl

${ }^{\dagger}$ Electronic address: lastowiecki@ift.uni.wroc.pl

${ }^{\ddagger}$ Electronic address: blaschke@ift.uni.wroc.pl are located in the QCD phase diagram is not accessible for terrestrial experiments or lattice simulations of QCD. The physics of NS is studied intensively in order to derive constraints for theories of high density physics aiming to complement the insights obtained from heavy-ion collisions. In the effort to understand the complex physics of neutron stars a large variety of observables has been studied which provide valu- 
able constraints on the equation of state (EoS). For recent reviews see, e.g., [2 6]. A crucial observable in this investigation is the maximum attainable mass of a NS which is directly connected to the EoS. The precise knowledge of the highest NS mass puts significant constraints on the stiffness of the EoS and can rule out entire classes of EoS models[7]. More advanced approaches aim to process simultaneously spectra, luminosities and distances of as many as possible NS in order to extract masses and radii simultaneously. These information can be further evaluated to determine a most probable underlying EoS within a Bayesian framework [8, 9]. While this method promises a detailed reconstruction of the EoS it relies on the availability of high quality, indisputable data suitable for the extraction of mass and radius constraints. Efforts are being made in the astronomers community to achieve this goal [10].

The recent measurement of two massive neutron stars with $M_{1614}=1.97 \pm 0.04 \mathrm{M}_{\odot}($ for PSR $\mathrm{J} 1614-2230$ [11]) and $M_{0348}=2.01 \pm 0.04 \mathrm{M}_{\odot}$ (for PSR J0348-0432 [12]) revived the discussion about possible implications of a NS with about two solar masses for the equation of state of cold and dense matter [7, 13]. These well measured masses greatly exceed the previously highest well known NS mass of $1.667 \pm 0.021 M_{\odot}$ for PSR J1903+0327 14, 15]. Because of the narrow error bands already this object provided a strong constraint on the stiffness of the equation of state. For example, it was suggested that this measurement puts a considerable strain on the possibility of the existence of quark matter in the cores of dense stellar objects [7]. A discussion similar to todays regarding PSR J1614-2230 followed when a pulsar mass of about $2 M_{\odot}$ had been reported for J0751+1807 [16], see [17] and references therein. Although this value had to be corrected afterwards, all conclusions drawn from the mere fact, that such heavy NS exist still stand. Contrary to other claims (e.g., Ref. [18]) we emphasize that observations of NS with masses of $2 M_{\odot}$ and beyond do not exclude the existence of hybrid NS with a quark matter core [19]. Partially, we use the present work to reemphasize this known fact within the framework of an Nambu-Jona-Lasinio (NJL) model in a similar but more detailed analysis as we performed it in previous work [17].

The appearance of new degrees of freedom, the most prominent being hyperons and quarks, entails a softening of the EoS and in general reduces the maximum NS mass in comparison to the underlying pure nuclear matter EoS. This softening, however, is not necessarily strong enough to conclude that NS masses as high as $2 M_{\odot}$ preclude the existence of exotic matter in the NS core. This has been confirmed in a number of studies concerning both, the occurrence of hyperons (see, e.g., 20-24]) and the transition to quark matter described within various different model approaches, e.g., [25 34]. The discussion of the limits on the stiffness of the high-density EoS is also performed in the context of heavy- 
ion collision experiments which provide further constraints on the EOS stiffness, see [34, 35].

In the present paper we systematically scan a part of the NJL model parameter space in order to locate those parameter regions which result in QM cores for massive NS. We fully scan the region of vector- and diquark couplings which result in stable NS configurations with QM cores. Additionally, we apply two different parameterizations regarding the scalar coupling, which both reproduce the pion and kaon mass as well as the pion decay constant and light quark mass in vacuum. The differences between them result from slightly different choices for the scalar coupling constant which are compensated by a different three-momentum cutoff $\Lambda$. Details concerning the parameterization scheme are found in [36]. Additionally, we investigate how these hybrid EoS which we find to favor massive NS with QM core agree with the flow constraint [37].

In order to keep this study sufficiently transparent we have chosen to vary the free parameters of the EoS model only in the quark sector and to apply the ab-initio Dirac-Brueckner-Hartree-Fock (DBHF) EoS using the Bonn-A nucleon-nucleon potential [38] as the only nuclear matter EoS we investigate. The latter well describes the saturation properties of symmetric nuclear matter, provides a sufficiently high maximum NS mass of $2.4 M_{\odot}$, and is in agreement with the flow constraint up to 3.5 times saturation density [39]. The phase transition between nuclear and quark matter is modelled in terms of a Maxwell construction.

This work is structured in the following way: Sect. II discusses the NJL model and a variety of coupling channels one could account for. While we do not consider all of these channels we find it instructive to discuss the variety NJL-type models offer. Later in the section we focus on the model as it is used for this study, namely accounting for the scalar-, vector-, and diquarkinteraction channel in the mean field approximation. In Sect. III we discuss the obtained compact star sequences and the agreement of the corresponding EoS parameterizations with flow data in symmetric matter. Section IV discusses our conclusions of this study.

\section{DENSE HYBRID STAR MATTER}

\section{A. NJL-type quark matter models}

The NJL model has originally been introduced by Nambu and Jona-Lasinio as a field theoretical model to understand the origin of the mass of nucleons as a selfenergy in a theory with four-fermion interactions in analogy to the occurrence of an energy gap in the theory of superconductivity [40]. Nowadays this model is widely appreciated as a useful tool to model the thermodynamics of deconfined quark matter, see [41] for a comprehensive review with particular emphasis on the high density aspects. Several reasons contribute to this fact. First, it describes already in a very simplified form, which 
takes only the attractive scalar interaction term into account, one of the most prominent key features of $\mathrm{QCD}$, the dynamical breaking of chiral symmetry. This is a clear distinction to the thermodynamic bag model which still is widely applied as it is easy to use for explorative purposes. A further advantage of NJL-type models is the availability of a wide number of different interaction channels which would result, e.g., from a global color model of QCD [42] after Fierz rearrangement of the current-current type interaction [43, 44]. Even though we strongly benefit from the NJL models 'simplicity' we point out, that it cannot be claimed to be equivalent to QCD. It reproduces some of the symmetries of the full theory, but not all. Among the missing features we mention the local color gauge symmetry. Further, interactions are considered on the level of one-gluon exchange. Hence, higher order non-perturbative self-interactions are ignored [45].

In this section we discuss the choices we made for the interaction part to define what we consider as a standard NJL model for applications to compact star physics. We also discuss aspects of NJL-type models which we denote as extensions to the standard NJL model.

Of importance in the low temperature domain at high densities is the formation of diquark condensates signalling color superconducting properties of the system. Solutions of the self-consistent meanfield equations for the quark masses and diquark gaps within the 3- flavor NJL model have first been presented in [46 48]. Shortly after, the before neglected vector channel interaction has been included [17]. This resulted in stiff QM EoS which describe hybrid NS with QM cores in full agreement with the observation of even the most massive NS given the nuclear matter EoS is sufficiently stiff [17].

The NJL Lagrangian for a quark matter model can be obtained from a global color model of QCD which ascribes all nonperturbative lowenergy QCD aspects to the coupling of quark currents via a model gluon propagator which in the limit of heavy gluon exchange reduce to a local coupling. Technically this is followed by a Fierz-transformation which results in a number of different interaction channels. The Fierztransformation gives an explicit ratio between the coupling constants of the different channels, which, if applied strictly, usually do not result in the very best results when it comes to describing NS observables. As it shows, already rather small variations of the couplings do significantly change the outcome of this kind of studies. Performing these variations seems completely legitimate to us, considering the before stated fact, that an NJL-model due to the underlying simplifications can at best be understood as an effective model, which consequently should be interpreted flexible enough to describe reality if adjusted properly. The interplay of all possible interaction channels so far has never been fully studied. We conclude at the current stage of re- 
search that one should systematically investigate the influence of every single one. Further terms, which do not directly follow from the sketched approach but can result from the full theory, as for instance the Polyakov-loop term describing the gluon sector, should be carefully added.

As a standard NJL-type model Lagrangian for dense quark matter studies we define

$$
\mathcal{L}=\mathcal{L}_{0}+\mathcal{L}_{S}+\mathcal{L}_{P S}+\mathcal{L}_{V}+\mathcal{L}_{A V}+\mathcal{L}_{D}
$$

The first term in the Lagrangian is the kinetic term for free Dirac quarks,

$$
\mathcal{L}_{0}=\bar{q}\left(-i \gamma^{\mu} \partial_{\mu}+\hat{m}+\gamma_{0} \hat{\mu}\right) q
$$

where $\hat{m}=\operatorname{diag}_{f}\left(m_{u}, m_{d}, m_{s}\right)$ is the current quark mass matrix, and $\hat{\mu}=\operatorname{diag}_{f}\left(\mu_{u}, \mu_{d}, \mu_{s}\right)$ the corresponding matrix for the quark chemical potentials. Instead of accounting explicitly for the minimal coupling to the non-Abelian, selfinteracting gluon fields prominent in the original QCD Lagrangian, we understand here that the gluon degrees of freedom are "integrated out" leaving instead an interaction Lagrangian consisting of combinations of quark bilinears with nonperturbatively strong couplings. The standard choice for these current-current interaction terms is guided by the observed meson spectrum and by chiral symmetry. The most important of these are the scalar and pseudo-scalar interaction channels,

$$
\begin{aligned}
\mathcal{L}_{S} & =G_{S} \sum_{a=0}^{8}\left(\bar{q} \tau_{a} q\right)^{2} \\
\mathcal{L}_{P S} & =G_{P S} \sum_{a=0}^{8}\left(\bar{q} \gamma_{5} \tau_{a} q\right)^{2}
\end{aligned}
$$

with the Gell-Mann matrices $\tau_{a}$ acting in flavor space, gives access to the key-feature for which NJL-type models are widely used, viz. the dynamical breaking of chiral symmetry. The pseudo-scalar channel does not contribute to the thermodynamical potential in meanfield approximation, but as it is formally necessary in order to keep the Lagrangian chirally invariant, we consider it being part of the model. Consequently, as soon as local variations of the scalar contributions are investigated, both terms should be taken into account. Chiral symmetry then requires $G_{P S}=G_{S}$.

The next two terms describe the vector and pseudo-vector interaction, again of currentcurrent type. As discussed earlier they are extremely important if one investigates the question whether QM occurs in the interior of NS. It has often been claimed that the existence of massive NS implies a negative answer. This statement, although wide spread and often met is nevertheless wrong. The reason for this wrong conclusion is usually found in a particular choice for the model which is supposed to describe QM at finite densities. This manifests in an insufficient stiffness of the EoS even though there is nor theoretical reason for such a limitation. In our model a stiffening is described by the repulsive vector (and pseudo-vector) interaction channel,

$$
\begin{aligned}
\mathcal{L}_{V} & =G_{V}\left(\bar{q} i \gamma_{\mu} q\right)^{2}, \\
\mathcal{L}_{A V} & =G_{A V}\left(\bar{q} i \gamma_{5} \gamma_{0} q\right)^{2} .
\end{aligned}
$$

Note, that these terms naturally appear after 
Fierz transformation of the local heavy gluon exchange interaction model which would then fix also the coupling strengths relative to the scalar channel as $G_{V}=G_{A V}=\frac{1}{2} G_{S}$ (see, e.g., appendix A of [41]). As for the pseudo-scalar interaction the term related to the axial-vector coupling vanishes in mean field approximation but is required to keep the Lagrangian chirally symmetric. In this sense we consider it to be part of the standard NJL model without impact on the mean field thermodynamics.

The last term we consider accounts for scalar diquark correlations,

$$
\mathcal{L}_{D}=G_{D} \sum_{a, b=2,5,7}\left(\bar{q} i \gamma_{5} \tau_{a} \lambda_{b} C \bar{q}^{T}\right)\left(q^{T} C i \gamma_{5} \tau_{a} \lambda_{a} q\right)
$$

where $\tau_{a}$ are again the Gell-Mann matrices in flavor space, but we also introduce their counterparts $\lambda_{a}$ acting in the color space. The matrix $C$ is the charge conjugation matrix and $G_{D}$ is yet another coupling constant introduced into the model. Note, that this term involves an interaction vertex of the $(q q)^{2}$ type and that it is antisymmetric w.r.t. quark exchange thus fulfilling the requirement of the Pauli principle for the diquark correlation. As for the vector coupling channel the diquark interaction channels can be obtained via Fierz transformation of the heavy gluon exchange model [41] which results in $G_{D}=0.75 G_{S}$. The scalar diquark interaction (7) is attractive and can therefore lead to diquark condensation at low temperatures and high densities, according to the Cooper theorem
[49]. As opposed to early works on diquark condensates and resulting color superconductivity which were based on perturbative one gluon exchange [50], the renaissance of color superconductivity started in 1997 was based on nonperturbative interaction models like the above NJL one (see, e.g., 51 53]) and gave large diquark pairing gaps of the order of the fermion mass. Therefore, the scalar diquark channel (7) gives important contributions to the thermodynamics of cold, dense quark matter and cannot be omitted in effective models of compact star matter. These terms describe the standard NJL model as we use it for the present study.

However, several extensions are possible. We want to name a few of them which we consider important. The first comes from the so called Kobayashi-Maskawa-'t Hooft (KMT) interaction $[54,55]$

$\mathcal{L}_{K}=-K\left[\operatorname{det}_{f}\left(\bar{q}\left(1+\gamma_{5}\right) q\right)+\operatorname{det}_{f}\left(\bar{q}\left(1-\gamma_{5}\right) q\right)\right]$

where the determinants are taken in flavor space. This determinant interaction is based on the single instanton solution and gives access to the $U_{A}(1)$ anomaly and the resulting $\eta-\eta^{\prime}$ mass splitting. However, there exist different sources for this symmetry breaking [56] which relate this aspect of low energy QCD to nonperturbative field configurations in the gluon sector which (unlike the instantons) are also related to confinement such as center vortices or a squeezed gluon condensate [57, 58]. To date, it is not clear 
which effects on the mean field thermodynamics of quark matter such alternative realizations of the $U_{A}(1)$ anomaly would have, if any. We consider any modeling of the $U_{A}(1)$ symmetry breaking such as the KMT interaction (and its Fierz transformed interaction [59 61] which involves the coupling of chiral and diquark condensates) as part of an extension beyond the standard NJL-type model introduced above. Second, we mention the Polyakov loop potential $U(\Phi, \bar{\Phi})$ which is often added to NJL-type model Lagrangians in order to account for the existence of gluons and the phenomenon of (de)confinement. In Polyakov gauge $\left(A_{4}=\phi_{3} \lambda_{3}+\phi_{8} \lambda_{8}\right)$ the fields $\Phi$ and $\bar{\Phi}$ can be expressed as

$$
\Phi=\frac{1}{N_{c}} \operatorname{Tr}_{c}\left\{\exp \left[i \int_{0}^{\beta} d \tau A_{4}(\tau)\right]\right\} .
$$

The form of the potential $U(\Phi, \bar{\Phi})$ in the presence of quarks, in particular at finite densities is not uniquely determined. Several potential ansätze have been proposed in the literature 62, 63] where the temperature dependence of its coefficients has been determined by pure gauge lattice QCD. A possible extension to finite chemical potentials has been proposed based on dimensionally consistent combinations of powers in terms of temperature and chemical potential [64, 65].

A third extension could be implemented by a residual bag pressure which may even be chemical potential dependent to account for possible medium dependences of the gluon sector, as from a "melting" of the gluon condensate. For recent models including a bag function along with a color superconducting NJL model of quark matter see, e.g., [33, 65 67].

Finally, we mention so-called crystalline (color) superconducting phases, also known as Larkin-Ovchinnikov-Fulde-Ferrell (LOFF) phases because of their similarity to condensed matter superconductors with magnetic impurities [68, 69], see Ref. [70] for a recent review. Initial investigations of LOFF phases [71, 72] were performed with fixed quark masses and thus ignored the fact that a simultaneous selfconsistent solution of light and strange quark mass gap equations together with the pairing gap equations is essential for the phase structure itself. This has been corrected later [73] and revealed that two-flavor color-superconducting LOFF phases can be energetically favored in compact stars and form stable hybrid star configurations with masses above $2 M_{\odot}[74,75]$. The crystallinity of the 2SC phase may not essentially affect the question for the maximum mass of hybrid stars but can affect their cooling behavior and therefore our understanding of NS phenomenology [76].

Let us now turn to the thermodynamical potential of the standard NJL model for QM under NS constraints in the mean field approximation [17, 46], 
$\Omega(T, \mu)=\frac{\phi_{u}^{2}+\phi_{d}^{2}+\phi_{s}^{2}}{8 G_{S}}-\frac{\omega_{u}^{2}+\omega_{d}^{2}+\omega_{s}^{2}}{8 G_{V}}+\frac{\Delta_{u d}^{2}+\Delta_{u s}^{2}+\Delta_{d s}^{2}}{4 G_{D}}-\int \frac{d^{3} p}{(2 \pi)^{3}} \sum_{n=1}^{18}\left[E_{n}+2 T \ln \left(1+e^{-E_{n} / T}\right)\right]+\Omega_{l}-\Omega_{0}$.

With $\Omega_{l}$ we added the lepton contributions (electrons and muons), $\Omega_{0}$ guarantees zero pressure in the vacuum. The extrema with respect to a variation of the meson and diquark mean fields $\phi_{f}, \omega_{f}$ and $\Delta_{f k}$ then define the gap equations

$$
\frac{\partial \Omega}{\partial \phi_{f}}=\frac{\partial \Omega}{\partial \omega_{f}}=\frac{\partial \Omega}{\partial \Delta_{f k}}=0
$$

their solutions determine thermodynamically stable equilibrium solutions.

\section{B. Model parameters}

As in-medium properties of quark matter are barely known, an appropriate strategy to adjust free model parameters is to describe well known vacuum properties of mesons (in general: hadrons). In our model this is possible for the scalar coupling strength $G_{S}$ and the momentum cut-off $\Lambda$ which is necessary to regularize the divergent one-loop integrals. More parameters which enter the model are the current quark masses $m_{u}, m_{d}$ and $m_{s}$. For simplicity we set $m_{u}=m_{d}$. In the chiral limit, for vanishing current quark masses, the pion and the kaon are true massless Goldstone bosons of the broken chiral symmetry. The two parameters $G_{S}$ and $\Lambda$ are then adjusted by the pion decay constant $f_{\pi}=93 \mathrm{MeV}$ and, e.g., the chiral condensate $\langle\bar{u} u\rangle=-(240 \mathrm{MeV})^{3}$. The light and strange current quark masses are then fixed by the pion and kaon mass, respectively. The details of this parameterization procedure and a number of representative parameterizations including the two sets used in the present work are found in [36] [82]. The fitting procedure is not without ambiguities regarding the relation between scalar coupling, light quark masses, remaining parameters and the hadron properties the model is adjusted to. In other words it is possible to describe this set of values with different values of the scalar coupling. Even though this does not give us arbitrary freedom to choose a scalar coupling, slight variations are possible.

The values of the vector coupling strength $G_{V}$ and the diquark coupling strength $G_{D}$ can be constrained by the values they attain if all interaction channels originated from the Fierz rearrangement of a heavy gluon exchange model. This defines their ratios to the scalar coupling strength $\eta_{V}=G_{V} / G_{S}$ and $\eta_{D}=G_{D} / G_{S}$ to be $\eta_{V}^{\text {Fierz }}=0.5$ and $\eta_{D}^{\text {Fierz }}=0.75$, respectively. These values actually result in a fair description of vector meson and nucleon masses (see, e.g., [44]). In this sense both values can be obtained from vacuum properties.

We take a slightly different perspective, based on the idea that both, vector meson and diquark interaction channels are particularly sus- 
ceptible to density effects and thus become strongly renormalized under dense matter conditions which we apply for this model. This situation is well known from the Walecka model for nuclear matter where the scalar and vector meson couplings are adjusted to the phenomenological saturation properties of nuclear matter rather than they reflect first principles.

Therefore, we use the above NJL model as an effective model with $\eta_{V}$ and $\eta_{D}$ being free parameters. A similar study we performed in [26] where we found hybrid EoS for a variety of $\eta_{V}$ and $\eta_{D}$ which would predict NS with a QM core.

In this study we extend this scan in order to explore the impact of the before mentioned ambiguity regarding the precise choice of the scalar coupling $G_{S}$. For this purpose we chose two parameterizations from Ref. [36] which both describe the same vacuum properties but differ by about $15 \%$ with respect to the scalar coupling. The resulting parameterizations are shown in Tab. I. Both sets have been used before, Set A to obtain the results of Ref. [77], Set B for example in Ref. [17]. Note, that the constituent quark mass of Set B is close to the nucleon mass divided by three and therefore close to what we would consider as a reasonable lower limit.

\section{Quark-hadron phase transition}

The NJL model as we use it describes the thermodynamics of deconfined quark fields and

\begin{tabular}{|c|c|c|c|c|c|}
\hline & $G_{S} \Lambda^{2}$ & $\Lambda[\mathrm{MeV}]$ & $m_{u}[\mathrm{MeV}$ & $m_{s}[\mathrm{MeV}]$ & $M_{u}[\mathrm{MeV}]$ \\
\hline & 2.319 & 602.30 & 5.500 & 112.00 & 367.5 \\
\hline et $B$ & 2.176 & 629.54 & 5.277 & 135.88 & 330.0 \\
\hline
\end{tabular}

TABLE I: NJL model parameterization used in the present study, taken from Ref. [36]. $M_{u}=m_{u}+\phi_{u}$ is the constituent light quark mass in vacuum.

does not account for the formation of hadrons due to confinement. A simple and feasible way to describe the transition from nuclear to quark matter is to take advantage of an independently calculated equation of state of nuclear matter. With this input the phase transition between the two phases is constructed based on the standard Gibbs rules of phase equilibrium. The nature of the QCD phase transition is not clear, there are ongoing discussions whether it is strongly first order (a Maxwell-like transition), permits the formation of regions with mixed phases of hadrons and quarks and even pasta-like phases or even a cross over transition with no critical end point in the QCD phase diagram at all. Here, we assume a strong first order phase transition, modelled by a Maxwell-construction. For details and the specifics of this construction under the constraints of $\beta$-equilibrium as well as electric and color charge neutrality in the presence of diquark condensates see, e.g., Refs. [78$80]$.

For our analysis we apply the nuclear DiracBrueckner Hartree-Fock (DBHF) EoS which has proven to perform reasonably well for describing nuclear matter saturation properties and kaon 
data [38] as well as NS properties [39] even though it tends to behave too stiff above densities of about 3.5 times saturation density. On the other side, this stiffness occurs in a region where QM degrees of freedom are not unlikely to be the only ones which are relevant. Amongst other reasons we prefer the DBHF EoS because it is based on a relativistic and microscopical description of many-particle interactions. It starts from a given free nucleon-nucleon interaction (the relativistic Bonn A potential) fitted to nucleonnucleon scattering data and deuteron properties. In ab initio calculations based on manybody techniques one then derives the nuclear energy functional from first principles, i.e., treating short-range and many-body correlations explicitly. In the relativistic DBHF approach the nucleon inside the medium is dressed by the selfenergy based on a T-matrix. The in-medium T-matrix as obtained from the Bethe-Salpeter equation plays the role of an effective two-body interaction which contains all short-range and many-body correlations in the ladder approximation. As we have shown in the context of hybrid EoS the rather stiff behavior at high densities is not necessarily relevant if the phase transition to QM occurs at low enough densities of about three to four times saturation density [17].

\section{RESULTS}

For both sets, A and B, with different parameterizations regarding the scalar coupling strengths $G_{S}$ we calculated the full QM EoS for eight values of the effective vector coupling $\eta_{V}$ between 0 and 0.7 in steps of 0.1 . For the effective diquark coupling we chose a stepwidth of 0.02 in the interval $[0.8,0.94]$ and 0.01 in the interval $[0.94,1.15]$ equal to 29 different values. These choices provided a sufficient coverage of the range of parameters which can result in stable hybrid star configurations. Additionally we performed the same amount of calculations for symmetric matter. This gives a total of 928 different QM EoS we computed, where each required about two hours of computing time on a $2.7 \mathrm{GHz}$ quad-core Opteron(tm) processor of which we had ten available.

With the resulting hybrid EoS we solved the TOV equations and obtained mass-radius (MR) and mass-central density relations (M-n) relations for spherically symmetric compact stars. The computational effort for this part has been negligible despite the very large number of calculated neutron stars.

In the following we explore the relation between the free parameters $\left(\eta_{D}, \eta_{V}\right.$, and the scalar coupling strength $G_{S}$ ) of the introduced NJL model EoS and the resulting NS characteristics (maximum NS masses, critical NS masses for the phase transition, and the corresponding $\mathrm{M}-\mathrm{R}$ relations). We will highlight the parameter regions where agreement with the observations of $2 M_{\odot}$ pulsars and flow measurements in HIC could been obtained. 


\section{A. Analysis of the $2 \mathrm{M}_{\odot}$ constraint}

In order to illustrate the general influence of the coupling constants $\eta_{D}$ and $\eta_{V}$ we will first vary only one of them and keep the other constant. We start with the diquark coupling at a constant value of $\eta_{D}=1.0$ and vary the vector coupling $\eta_{V}$. As it is expected from an repulsive interaction channel increasing $\eta_{V}$ increases the stiffness of the QM EoS. As a consequence a higher value of $\eta_{V}$ leads to higher densities for the onset of the phase transition and simultaneously to higher maximum NS masses. The same general behavior holds for both sets, A and B, as we illustrate in Figs. 1 and 2. Note, that despite
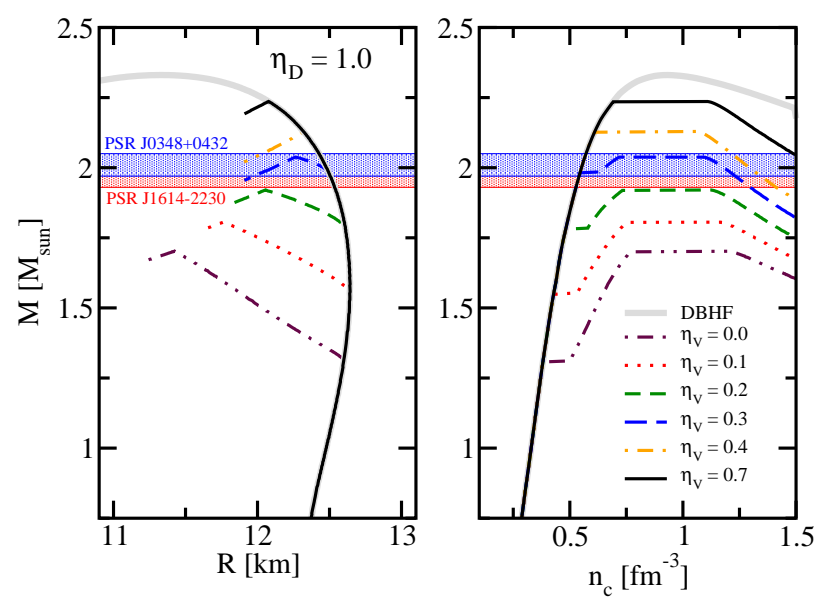

FIG. 1: (Color online.) Mass-radius and masscentral density sequences for varying vector coupling strength $\eta_{V}$ at fixed $\eta_{D}=1.0$ for set A.

this general stiffening of the EoS with increasing vector coupling, the transition densities for the same sets of values $\left(\eta_{D}, \eta_{V}\right)$ differ between both sets. This difference is most pronounced at small
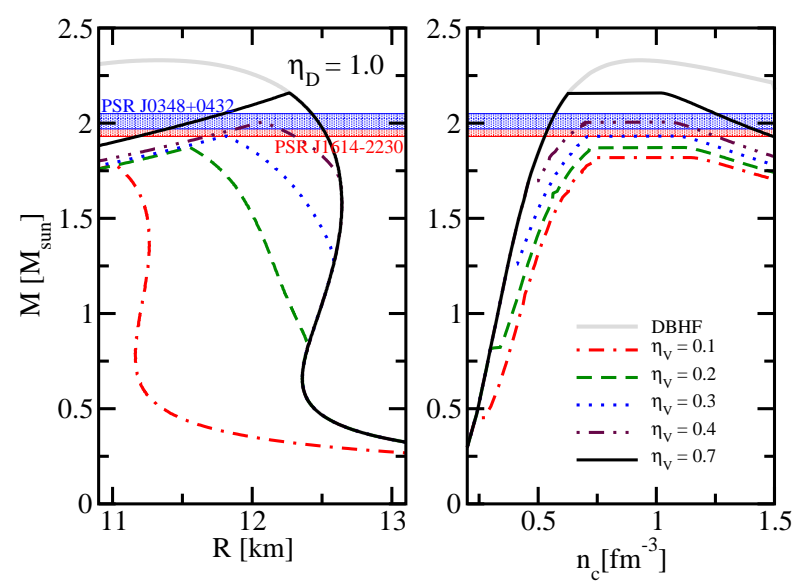

FIG. 2: (Color online.) Same as Fig. 1 for set B.

values of $\eta_{D}$ where Set A shows an onset of QM at higher densities than Set B.

Next, we perform a variation of $\eta_{D}$ at a fixed value of $\eta_{V}=0.3$. Again set $\mathrm{A}$ and $\mathrm{B}$ have the same systematic behavior with only quantitative differences, as shown in the Figs. 3] and 4. Increasing the coupling strength in the diquark interaction channel lowers the critical density and at the same time the maximum attainable mass is lowered due to the resulting softening of the EoS.

Comparing set A and B at the same values of parameters $\eta_{D}$ and $\eta_{V}$ shows that a lower constituent quark mass (set B) results in an earlier transition to QM. We point out that because both, $\eta_{D}$ and $\eta_{V}$, are defined as the ratio of the corresponding coupling strengths to the scalar coupling strength $G_{S}$ we explicitly do not compare equal coupling strengths in the coupling channels.

In Figs. 5 and 6 we present M-R curves for 
would have to increase also the diquark coupling
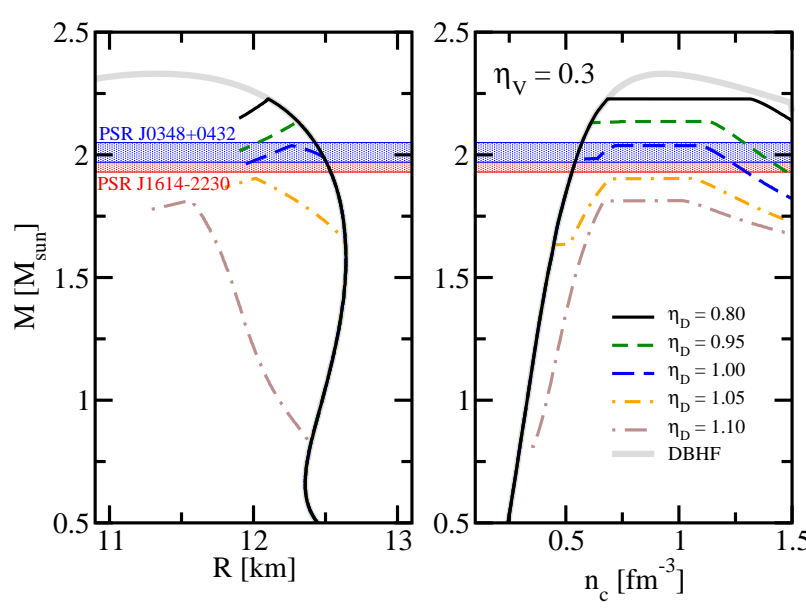

in order to keep the maximum NS mass at a constant value. This represents the earlier stated fact, that $\eta_{V}$ stiffens and $\eta_{D}$ softens the EoS. Therefore, we find that the constraint of a given NS mass is fulfilled for monotonously rising functions in the $\eta_{D}-\eta_{V}$ parameter plane.

We note, that for any hybrid EoS which reproduces the maximum mass of PSR J1614-2230 the radius of the most massive configuration

FIG. 3: (Color online.) Mass-radius and masscentral density sequences for varying diquark coupling strength $\eta_{D}$ at fixed $\eta_{V}=0.3$ for set A.
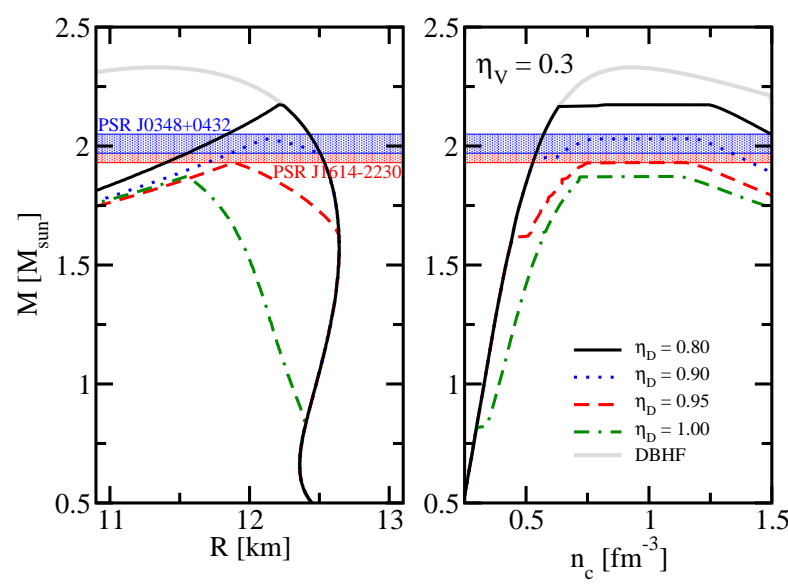

FIG. 4: (Color online.) Same as Fig. 3 for set B.

parameter sets chosen such that the maximum obtained mass is $1.97 M_{\odot}$, corresponding to the mass expectation value of PSR J1614-2230 [11] and to the lower limit of the $1 \sigma$ band of the mass measurement for PSR J0348+0432 [12].

One clearly observes the general tendency that when increasing the vector coupling one does not depend significantly on the chosen parameters and is around $12 \mathrm{~km}$.
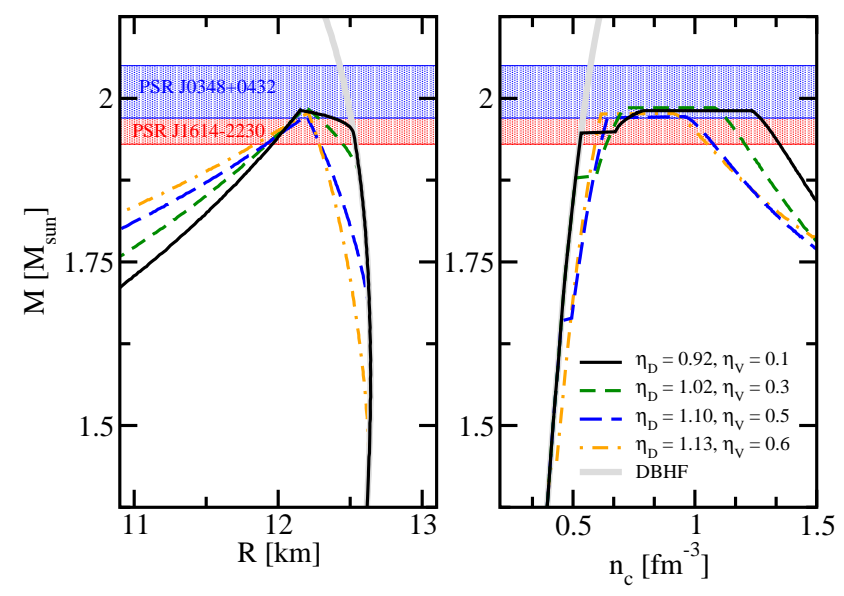

FIG. 5: (Color online.) Systematics of mass-radius and mass-central density curves for set $\mathrm{A}$ with the parameter pairs $\left(\eta_{D}, \eta_{V}\right)$ chosen such that the maximum mass equals $1.97 M_{\odot}$, the mass of PSR J16142230 .

The discussed results are summarized in Fig. 7 and Fig. 8 for Set $\mathrm{A}$ and Set B, respectively. The red band covers all possible parameterizations which result in a maximum mass equal to the mass of PSR J1614-2230 while 


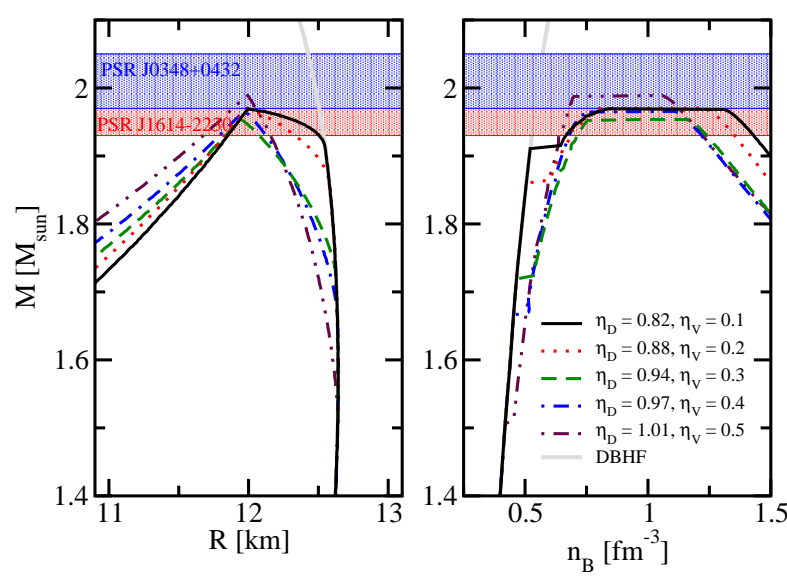

FIG. 6: (Color online.) Same as Fig. 5 for set B.

tain any stable hybrid NS configuration. Even though all these solutions are purely hadronic, the maximum masses in this region can differ, as the phase transition to quark matter can occur at central densities below the central density corresponding to the maximum mass of the purely hadronic EoS. In this case a transition to QM only results in a lowering of the maximum mass of purely hadronic NS due to the instability of the hybrid star configurations. The light orange region denoted as "Quark Stars" deserves a separate discussion. In this domain we find quark

the blue band corresponds to the mass of PSR J0438+0432. Non-solid black lines refer to a certain NS mass (given in the legend) at which QM appears in the NS core. This information is useful to estimate the amount of QM in a given parameterization. Note, that if one follows the red (or blue) band from the left to the right the corresponding critical NS mass, where QM appears first, decreases. This just illustrates that one can expect larger QM cores in NS configurations with higher values of $\eta_{D}$ (and consequently $\eta_{V}$ ) if the different EoS all result in the same maximum NS mass. Of course, the reason for this is a lowering of the critical density along the red (blue) band from the left to the right. The solid black line, labelled with $2.1 M_{\odot}$ denotes the border between model parameter regions for which the maximum NS mass is below or beyond $2.1 M_{\odot}$.

At any given $\eta_{D}$, heavier configurations are found at higher values for $\eta_{V}$. For parameterizations within the cyan region we do not obmatter favored over hadronic matter at all densities (or chemical potentials). Therefore, a phase transition does not occur. Even though we consider the total missing of a phase transition, in particular at low densities, as an artefact of our QM model one can conclude, that in this domain the phase transition is taking place at extremely low densities. Therefore, the label 'quark stars' is justified even though the distinction between hybrid configurations and pure quark stars is not as strict as it appears. Near this border we observe a phenomenon known as masquerade effect [81] where the EoS for quarks and hadrons are nearly identical and one therefore obtains almost identical M-R curves (hence the masquerade) for the purely hadronic and the hybrid EoS. In this scenario one can sometimes observe multiple crossings of the hadronic and QM EoS in the $\mathrm{P}-\mu$ plane. This can be interpreted as an indicator for a crossover transition from one phase to the other, characterized by identical EoS in 
the transition region. Certainly, a Maxwell construction as we have performed is not suited to address this scenario.

The green lines in Figs. 7 and 8 refer to the critical density of symmetric hadronic matter where the phase transition to QM takes place (the values are given in the plot). As this concerns properties of symmetric matter we will discuss this in more detail in the following section.

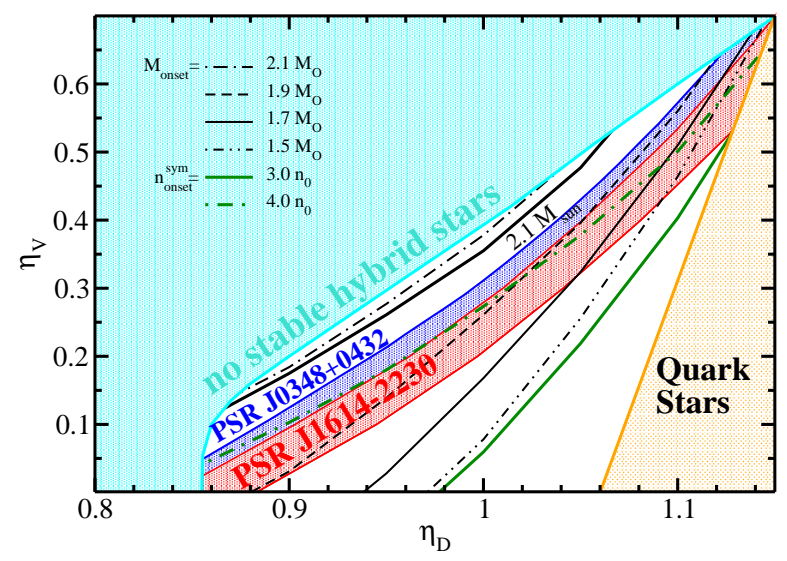

FIG. 7: (Color online.) Full analysis of hybrid NS with QM core in the $\eta_{V}-\eta_{D}$ parameter space for set A. The colored hatched regions denote parameter pairs where no stable hybrid stars are possible (cyan), the entire star is composed of quark matter (orange) and where the maximum mass for the hybrid EoS is contained in the $1 \sigma$ band of the mass measurement for PSR J1614-2230 (red) or PSR J0438+0432 (blue). The bold green lines denote given densities for the QM onset in symmetric matter: $3 n_{0}$ (solid) and $4 n_{0}$ (dash-dotted). The thin black lines stand for given NS masses at QM onset: $2.1 M_{\odot}$ (dash-dotted), $1.9 M_{\odot}$ (dashed), $1.7 M_{\odot}$ (solid), and $1.5 M_{\odot}$ (dashdouble dotted).

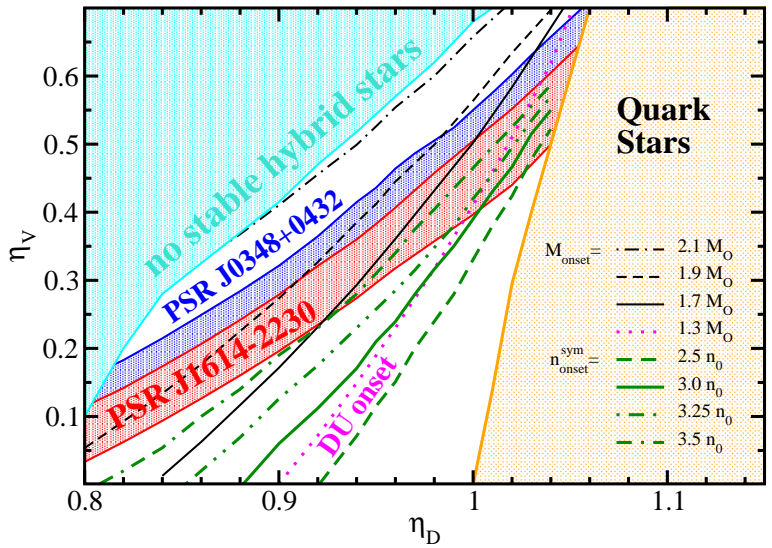

FIG. 8: (Color online.) Same as Fig. 7 for Set B; additional densities for the QM onset in symmetric matter (see legend) indicate the onset of the direct Urca (DU) cooling process in NS matter at $1.3 \mathrm{M}_{\odot}$ according to the hadronic DBHF EoS (bold dotted magenta line).

\section{B. Connection to symmetric matter and the flow constraint}

While matter in a NS due to the established $\beta$-equilibrium and electric charge neutrality is in general highly isospin asymmetric, in particular in the NS core, the matter in HIC is fairly isospin symmetric. Understanding the properties of symmetric matter under the HIC conditions of high temperature and density is of great importance for the exploration and the understanding of a wide range of phenomena. Focussing on the QCD phase transition one can divide measured observables roughly into those which are are highly sensitive to a phase transition and those which are not. Most observables 
concerning particle yields fall into the latter category. The most promising for the exploration of thermodynamic properties of matter in heavy ion collisions are processes associated with the hydrodynamic expansion of the fireball and connected to anisotropies in the observed particle distributions. An interpretation of the measurements in terms of thermodynamic properties is a difficult and highly involved task that suffers from systematic uncertainties. Attempts have been made to analyze elliptic flow data in order to specify a region in the pressure-density plane which provides upper and lower limits for the pressure at a given density [37].

Our conclusions for the qualitative dependence of the deconfinement phase transition on the $\eta$-parameters of our model hold in the same way for symmetric matter as discussed in the previous section for NS matter. We remind, that a higher value of $\eta_{V}$ leads to an onset of quark matter at higher densities and conversely a higher value of $\eta_{D}$ leads to an earlier transition. An increase of the constituent quark mass (by a larger scalar coupling strength $G_{S}$ ) leads to lower transition densities. Figs. 9 and 10 (for set A and B, respectively) show the EoS for symmetric matter at a fixed value of $\eta_{V}=0.3$ with different coupling strengths in the diquark channel. The results are plotted on top of the flow constrained region (green area). It is clearly visible that the red solid plotted hadronic DBHF EoS would violate the flow constraint at about $n=0.55 \mathrm{fm}^{-3}$, corresponding to 3.5 times the saturation density $n_{0}$. While describing the phase transition by performing a Maxwell construction the occurrence of a quark branch in the hybrid EoS necessarily leads to a softening of the EoS in the corresponding domain. In our scenario this turns out to be of advantage, as the violation of the flow constraint by $\mathrm{DBHF}$ at high densities is corrected for the hybrid EoS, given the phase transition occurs around the density $n \simeq 0.55 \mathrm{fm}^{-3}$. In this particular example with $\eta_{V}=0.3$ this holds for $\eta_{D} \gtrsim 1.05$ (Set A, Fig. 9) or $\eta_{D} \gtrsim 0.95$ (Set B, Fig. 10), respectively.

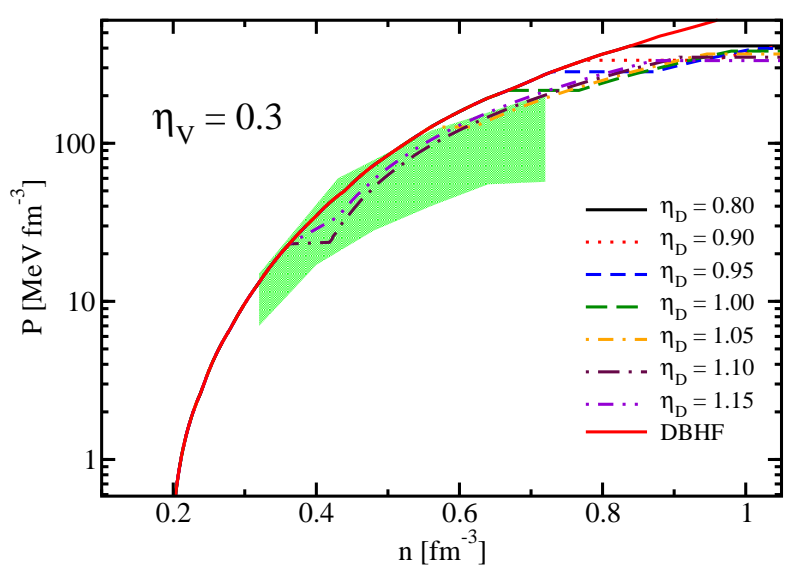

FIG. 9: (Color online.) Symmetric hybrid EoS at different $\eta_{D}$ for fixed $\eta_{V}=0.3$ in comparison to the flow constraint for Set A.

While the value of $\eta_{V}$ in the previous paragraph has been chosen arbitrarily one could ask for a justification of this parameter choice. As our aim is to find connections between NS and HIC observables we discuss the question what consequences arise for the phase transition in HIC if one would know that QM exists at least 


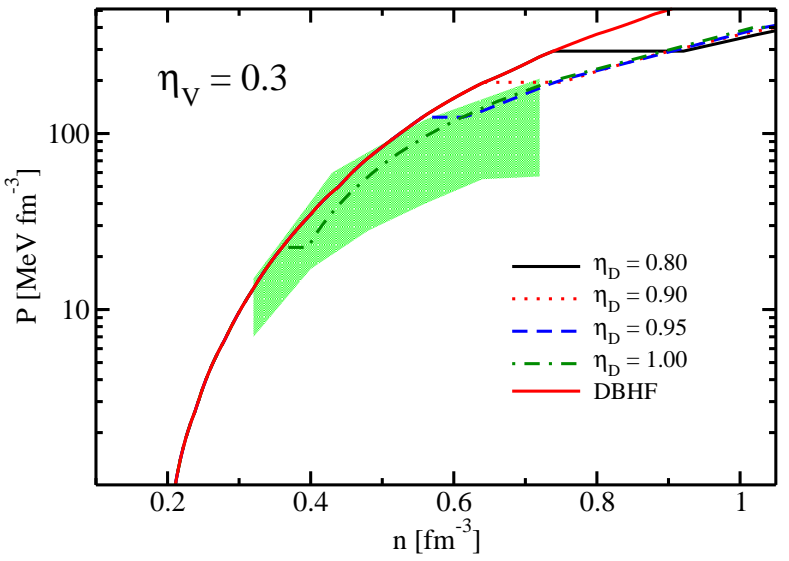

FIG. 10: (Color online.) Same as Fig. 9 for Set B.

in the heavier compact stars. Because the NS mass increases with the central density the opposite scenario - QM exists only in less massive NS - is not realistic for regular NS. This might be different for '3rd family' stars which arise from twin solutions where one can find a second stable branch of exotic NS with smaller radii and masses below those we discuss.

In Fig. 11 and Fig. 12 (representing Set A and B, respectively) we show symmetric EoS with parameters which under NS constraints result in a maximum masses of $1.97 M_{\odot}$, the mass of PSR J1614-2230. In both cases we find similar values for the critical density, all in the vicinity of the density where the DBHF EoS would begin to violate the flow constraint. For Set B, the parameterization with a smaller value of the constituent quark mass, the phase transition is shifted towards slightly lower densities. As this brings Set B into better agreement with the flow constraint, this slight change is meaningful. In

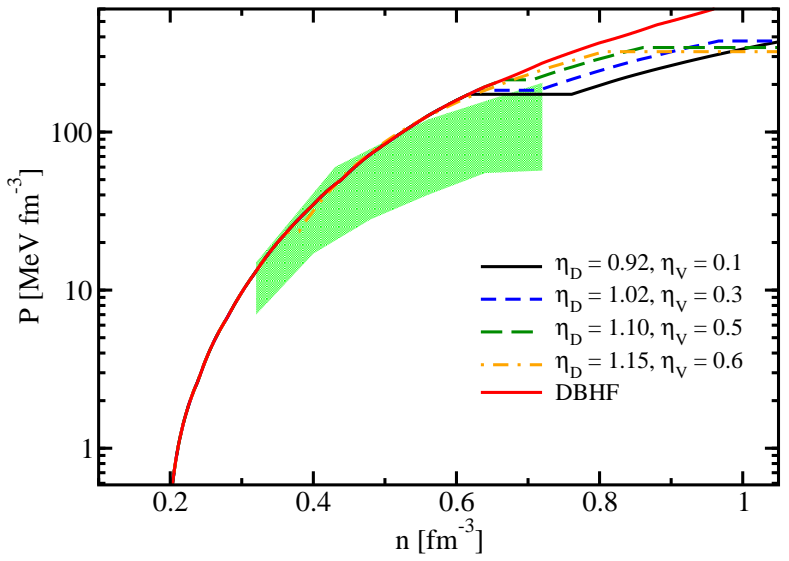

FIG. 11: (Color online.) Symmetric hybrid EoS for different pairs of $\left(\eta_{V}, \eta_{D}\right)$, all describing a maximum NS mass equal to that of PSR J1614-2230. (Set A.)

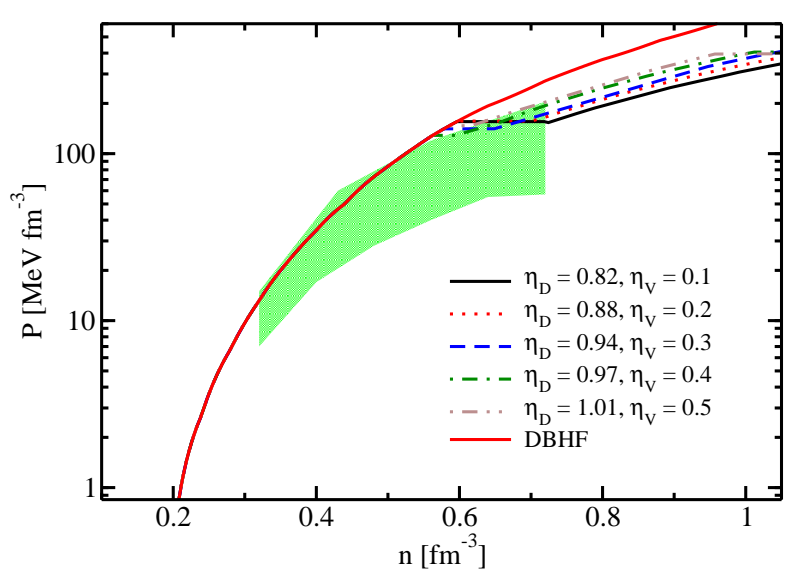

FIG. 12: (Color online.) Same as Fig. 11 for Set B.

these Figures the phase transition in symmetric matter appears to take place at an almost constant value. As the EoS parameterizations with respect to $\eta_{V}$ and $\eta_{D}$ are very different and just agree in reproducing the same maximum mass (chosen to be $1.97 M_{\odot}$, compatible with the measured masses of both $2 M_{\odot}$ pulsars, PSR J16142230 [11] and PSR J0438+0432 [12], within the 
$1 \sigma$ range) this gives the following interesting result.

If it turns out, that the mass of PSR J16142230 is close to the maximum mass a NS can maintain, then the actual parameters of the QM model are of minor relevance for the approximate value of the critical density in symmetric matter. This holds under the assumption that QM does exist in the core of this pulsar. If we assume the opposite, claiming that QM does not exist in any NS, our observation is still useful as the almost parameter independent critical density we found can be interpreted as the absolute lower limit to the actual critical density in symmetric matter. The phase transition in HIC under no circumstances can occur at lower densities as this would result in NS with quark matter core.

However, more valuable from the perspective of how to constrain our QM model is the first situation, where at least some NS are hybrid stars. If confirmed, this would greatly constrain the possible parameter range of our model. To illustrate this we refer to Fig. 7 and Fig. 8. In both cases the allowed region of parameters is limited from below by the lower edge of the red band and from above by the lower edge of the region with no stable hybrid solutions. If the existence of QM in compact stars is excluded by whatever reason a realistic QM EoS can, of course, be found only in the cyan hatched region of parameterizations which result in unstable hybrid configurations and configurations with a quark matter onset beyond the central density for the maximum mass of the purely hadronic EoS. Looking closely, one realizes that this argumentation works best for Set A. In the corresponding Fig. 7 the red (blue) band, denoting configurations with maximum masses in the $1 \sigma$ band of mass measurements for the $2 M_{\odot}$ pulsar PSR J1614-2230 (PSR J0438+432) runs almost parallel to the dash-dotted green curve which represents parameterizations with a constant critical density of $n_{c}=4.0 \mathrm{n}_{0}$. For Set B in Fig. 8 a similar discussion could hold for smaller values of $\eta_{D}$ (and therefore $\left.\eta_{V}\right)$. However, for values close to $\eta_{D}=1.0$ one can find very different critical densities in symmetric matter for slightly different parameterizations.

We stated early in this paper, that one of the big unknowns in our analysis is the hadronic EoS. In this sense our analysis is not complete and should be repeated with a wide range of different nuclear EoS in order to get a better idea about the possible spectrum of hybrid EoS obtained within the class of two-phase models with basic superconducting NJL quark matter. For now, we can just pretend, that DBHF is a fairly realistic hadronic EoS. A last, and very interesting result has to be understood from this perspective. If we strictly require that our QM EoS has to repair the violation of the flow constraint by DBHF this means that the phase transition in symmetric matter has to take place below a density of about $3.25 n_{0}$. In the case of Set $\mathrm{A}$ as visible in Fig. 7 this is difficult to achieve but not impossible. There is a small region at $\eta_{D} \approx 1.1$, 
$\eta_{V} \approx 0.55$ which would permit this. In this region of the parameter space, the size of the QM core in NS is large, the transition density consequently very low. If DBHF is a realistic EoS for low densities in NS matter and up to $3.25 n_{0}$ in symmetric matter and if the upper bound of the flow constraint is reliable the existence of heavy NS with QM content provides a very tight constraint on both, $\eta_{D}$ and $\eta_{V}$. The situation is a bit less extreme for Set B, as seen in Fig. 7 . In this case one would expect $\eta_{D}$ to be larger than 0.975 but not larger than 1.05 . The vector coupling $\eta_{V}$ in this domain can vary widely between about 0.4 and 0.6 .

We point out again, that Set A corresponds to higher constituent quark masses than Set B. Constituent quark masses below these for Set $\mathrm{B}$ are barely realistic. In this sense we find, that high constituent quark masses are less easily brought into agreement with DBHF as an EoS which does not fulfil the flow constraint. Ignoring this it seems easier for the same set to derive lower limits for the onset of quark matter in HIC.

\section{CONCLUSIONS}

The major conclusion of this investigation is that the existence of quark matter in neutron stars is not excluded by any current data. We described a wide number of parameterizations which result in NS configurations with a quark matter core that reach masses up to $2.1 M_{\odot}$ and thus fulfill the constraint from the new mass measurements of PSR J1614-2230 and PSR J0438+432 Some of our parameterizations resulted in NS with quark cores containing half of the total mass of the star. The biggest unknown in our investigation is the hadronic EoS which determines the maximum NS mass and the precise value of any quantity we investigated.

For one of the investigated sets (Set A) we found a strong correlation between the onset of QM in symmetric matter and the maximum mass of the hybrid star sequence. This particular model set up implies, that the critical density in symmetric matter is larger than or equal to four times saturation density. With our choice of the hadronic EoS (DBHF) this implies a violation of the flow constraint unless we choose the above mentioned set of very strong couplings $\left(\eta_{D}, \eta_{V}\right)=(1.1,0.55)$. If there is quark matter in neutron stars the transition density cannot be much larger because otherwise hybrid NS configurations turn unstable. On the other hand, even if there is no QM in NS the onset density cannot be lower, as this would theoretically result in stable hybrid configurations.

If heavy-ion collision experiments provide precise information about the transition density in symmetric matter, one can favor or disfavor the existence of quark matter in NS cores. This correlation is less distinct for Set B with different scalar coupling and a very small resulting constituent quark mass. In this case we still estimate the lowest transition density in symmetric matter to have a value of about $2.5 n_{0}$. However, 
a similar precise statement about the largest critical density which could still be in agreement with the hypothetical existence of QM cores in NS is out of scope considering the uncertainties due to the badly known hadronic EoS. Assuming, that DBHF is 'the' EoS and that a transition to QM repairs the violation of the flow constraint the upper limit is the density where this violation begins, namely $3.25 n_{0}$.

To bring NS phenomenology and HIC flow data in agreement high values of the diquark and scalar coupling are required. In particular the vector interaction channel should have a coupling value of $\eta_{V} \approx 0.5$ or above. For the diquark channel values of $\eta_{D}$ around 1.0 result in hybrid stars configurations which are in good agreement with our data.

A lower constituent quark mass leads to an earlier onset of quark matter. In this context such parameterizations favor the existence of QM in NS. The fulfillment of the flow constraint is easier to obtain with a smaller constituent quark mass. However, the outcome of this analysis depends strongly on the hadronic EoS. Ignoring the violation of the flow constraint which is induced to this analysis by the DBHF EoS allows a wide range of $\eta_{D}$ and $\eta_{V}$ for both, Set A and Set B. Both coupling parameters, $\eta_{V}$ and $\eta_{D}$, are correlated due to observed high mass NS.

Summarizing our conclusions, we find that the observation of the $2 M_{\odot}$ pulsars PSR J16142230 and PSR J0438+0432 provides strong constraints for the occurrence of quark matter in compact stars. Within chiral quark models of the NJL type the occurrence of QM in NS requires that QM must be color superconducting with a rather large diquark coupling constant and requires repulsive forces coming from a non vanishing mean field in the vector meson interaction channel.

These conclusions are drawn from a meanfield description of quark matter within a twophase description of quark-hadron hybrid matter. This analysis is not based on first principles but on the other hand represents what is the current state of the art concerning the modelling of matter at high densities and low temperature.

\section{Acknowledgements}

This work was supported in part by "CompStar" a research networking programme of the European Science Foundation and by a grant from the Polish Ministry for Science and Higher Education (MNiSW) supporting it. T.K. and D.B. acknowledge partial support by Narodowe Centrum Nauki (NCN) within the "Maestro" programme under contract No. DEC-2011/02/A/ST2/00306. Further support came from the Russian Fund for Basic Research under grant number 11-02-01538a (D.B.), the EU framework programme FP7 "hadronphysics3" (T.K.), a research grant number 2291/M/IFT/12 from the Faculty of Physics and Astronomy at the University of Wroclaw and by European Social Fund under project 
number POKL.04.01.01-00-054/10-00(R.Ł.).

[1] F. Weber, "Pulsars as astrophysical laboratories for nuclear and particle physics," Bristol, UK: IOP (1999)

[2] D. Blaschke, T. Klähn and F. Weber, in: Strongly Interacting Matter - The CBM Physics Book,

Lecture Notes in Physics 814, Springer (2010), pp. $158-181$.

[3] M. C. Miller, Nucl. Phys. A698, 233 (2002).

[4] S. Bhattacharyya, Advances in Space Research, 45, 949 (2010).

[5] J. Trümper, Prog. Part. Nucl. Phys. 66, 674 (2011).

[6] J. M. Lattimer, Ann. Rev. Nucl. Part. Sci. 62, 485 (2012) arXiv:1305.3510 [nucl-th]].

[7] J. M. Lattimer, M. Prakash, arXiv:1012.3208 [astro-ph.SR]].

[8] A. W. Steiner, J. M. Lattimer and E. F. Brown, Astrophys. J. 722, 33 (2010)

[9] A. W. Steiner, J. M. Lattimer and E. F. Brown, Astrophys. J. 765, L5 (2013)

[10] Z. Arzoumanian, S. Bogdanov, J. Cordes, K. Gendreau, D. Lai, J. Lattimer, B. Link, A. Lommen et al., astro2010: The Astronomy and Astrophysics Decadal Survey, 6, (2010).

[11] P. Demorest, T. Pennucci, S. Ransom, M. Roberts, J. Hessels, Nature 467, 1081 (2010).

[12] J. Antoniadis et al., Science 340, 6131 (2013).

[13] F. Özel, D. Psaltis, S. Ransom, P. Demorest, M. Alford, Astrophys. J. 725, 1918 (2010).

[14] D. J. Champion et al., Science 320, 1390 (2008).

[15] P. C. C. Freire, C. G. Bassa, N. Wex, I. H. Stairs, D. J. Champion, S. M. Ransom, P. Lazarus and
V. M. Kaspi et al., Mon. Not. Roy. Astron. Soc. 412, 2763 (2011).

[16] D. J. Nice, E. M. Splaver, I. H. Stairs, O. Loehmer, A. Jessner, M. Kramer, 2 and J. M. Cordes, Astrophys. J. 634, 1242 (2005).

[17] T. Klähn, D. Blaschke, F. Sandin, C. Fuchs, A. Faessler, H. Grigorian, G. Röpke, J. Trümper, Phys. Lett. B654, 170 (2007).

[18] F. Özel, Nature 441, 1115 (2006).

[19] M. Alford, D. Blaschke, A. Drago, T. Klähn, G. Pagliara and J. Schaffner-Bielich, Nature 445, E7 (2007).

[20] S. Weissenborn, D. Chatterjee and J. SchaffnerBielich, Nucl. Phys. A 881, 62 (2012).

[21] I. Bednarek, P. Haensel, J. L. Zdunik, M. Bejger and R. Manka, A\%A, 543, A157 (2012).

[22] A. Sulaksono and B. K. Agrawal, arXiv:1209.6160 [nucl-th].

[23] T. Katayama, T. Miyatsu and K. Saito, Nuclear Physics A, 895, 44 (2012).

[24] R. Łastowiecki, D. Blaschke, H. Grigorian and S. Typel, Acta Phys. Polon. Supp. 5, 535 (2012).

[25] S. Weissenborn, I. Sagert, G. Pagliara, M. Hempel and J. Schaffner-Bielich, Astrophys. J. 740, L14 (2011).

[26] T. Klähn, D. Blaschke and R. Łastowiecki, Acta Phys. Polon. Supp. B 5 (2012) 757.

[27] C. H. Lenzi and G. Lugones, Astrophys. J. 759 (2012) 57.

[28] N. Chamel, A. F. Fantina, J. M. Pearson and S. Goriely, Astron. Astrophys. 553 (2013) A22. arXiv:1205.0983 [nucl-th].

[29] B. Franzon, D. A. Fogaca, F. S. Navarra and J. E. Horvath, Phys. Rev. D 86 (2012) 065031. 
[30] K. Kim, H. K. Lee and M. Rho, Int. J. Mod. Phys. Conf. Ser. 10, 123 (2012).

[31] R. Mallick, Phys. Rev. C 87 (2013) 025804.

[32] K. Masuda, T. Hatsuda and T. Takatsuka, Astrophys. J. 764 (2013) 12.

[33] L. Bonanno and A. Sedrakian, Astron. Astrophys. 539, A16 (2012).

[34] I. Sagert, L. Tolos, D. Chatterjee, J. SchaffnerBielich and C. Sturm, Phys. Rev. C 86, 045802 (2012)

[35] T. Klähn, D. Blaschke, F. Weber, Phys. Part. Nucl. Lett. 9, 484 (2012).

[36] H. Grigorian, Phys. Part. Nucl. Lett. 4, 223 (2007).

[37] P. Danielewicz, R. Lacey, W. G. Lynch, Science 298, 1592 (2002).

[38] C. Fuchs, Lect. Notes Phys. 641, 119-146 (2004).

[39] T. Klähn, D. Blaschke, S. Typel, E. N. E. van Dalen, A. Faessler, C. Fuchs, T. Gaitanos, H. Grigorian et al., Phys. Rev. C74, 035802 (2006).

[40] Y. Nambu, G. Jona-Lasinio, Phys. Rev. 122 (1961) 345-358.

[41] M. Buballa, Phys. Rept. 407, 205 (2005).

[42] P. C. Tandy, Prog. Part. Nucl. Phys. 39, 117 (1997).

[43] R. T. Cahill, Austral. J. Phys. 42, 171 (1989).

[44] D. Ebert, H. Reinhardt and M. K. Volkov, Prog. Part. Nucl. Phys. 33, 1 (1994).

[45] S. P. Klevansky, Rev. Mod. Phys. 64, 649 (1992).

[46] D. Blaschke, S. Fredriksson, H. Grigorian, A. M. Oztas and F. Sandin, Phys. Rev. D 72, 065020 (2005).

[47] S. B. Ruester, V. Werth, M. Buballa, I. A. Shovkovy and D. H. Rischke, Phys. Rev.
D 72, 034004 (2005).

[48] H. Abuki and T. Kunihiro, Nucl. Phys. A 768, 118 (2006).

[49] L. N. Cooper, Phys. Rev. 104, 1189 (1956).

[50] D. Bailin and A. Love, Phys. Rept. 107, 325 (1984).

[51] R. Rapp, T. Schfer, E. V. Shuryak and M. Velkovsky, Phys. Rev. Lett. 81, 53 (1998).

[52] M. G. Alford, K. Rajagopal and F. Wilczek, Phys. Lett. B 422, 247 (1998).

[53] D. Blaschke and C. D. Roberts, Nucl. Phys. A 642, 197 (1998).

[54] G. 't Hooft, Nucl. Phys. B 61 (1973) 455.

[55] M. Kobayashi and T. Maskawa, Prog. Theor. Phys. 44, 1422 (1970).

[56] R. Alkofer, C. S. Fischer and R. Williams, Eur. Phys. J. A 38, 53 (2008).

[57] D. Blaschke, H. P. Pavel, V. N. Pervushin, G. Ropke and M. K. Volkov, Phys. Lett. B 397, 129 (1997).

[58] H. P. Pavel, D. Blaschke, V. N. Pervushin and G. Ropke, Int. J. Mod. Phys. A 14, 205 (1999).

[59] A. W. Steiner, Phys. Rev. D 72, 054024 (2005).

[60] T. Hatsuda, M. Tachibana, N. Yamamoto and G. Baym, Phys. Rev. Lett. 97, 122001 (2006).

[61] H. Abuki, G. Baym, T. Hatsuda and N. Yamamoto, Phys. Rev. D 81, 125010 (2010).

[62] S. Roessner, C. Ratti, W. Weise, Phys. Rev. D75 (2007) 034007.

[63] C. Ratti, M. A. Thaler and W. Weise, Phys. Rev. D 73, 014019 (2006).

[64] V. A. Dexheimer and S. Schramm, Nucl. Phys. Proc. Suppl. 199, 319 (2010).

[65] D. Blaschke, J. Berdermann, R. Łastowiecki, Prog. Theor. Phys. Suppl. 186, 81 (2010).

[66] R. Lastowiecki, D. Blaschke and J. Berdermann, Phys. Atom. Nucl. 75, 893 (2012). 
[67] G. Pagliara and J. Schaffner-Bielich, Phys. Rev. D 77, 063004 (2008).

[68] A. I. larkin and Y. N. Ovchinnikov, Zh. Eksp. Teor. Fiz. 47, 1136 (1964) [Sov. Phys. JETP 20, $762(1965)]$.

[69] P. Fulde and R. A. Ferrell, Phys. Rev. 135, A550 (1964).

[70] R. Anglani, R. Casalbuoni, M. Ciminale, R. Gatto, N. Ippolito, M. Mannarelli and M. Ruggieri, arXiv:1302.4264 [hep-ph].

[71] M. G. Alford, J. A. Bowers and K. Rajagopal, Phys. Rev. D 63, 074016 (2001) hep-ph/0008208.

[72] J. A. Bowers and K. Rajagopal, Phys. Rev. D 66, 065002 (2002) hep-ph/0204079.

[73] N. D. Ippolito, G. Nardulli and M. Ruggieri, JHEP 0704, 036 (2007) hep-ph/0701113.

[74] R. Anglani, R. Gatto, N. D. Ippolito, G. Nardulli and M. Ruggieri, Phys. Rev. D 76, 054007 (2007) arXiv:0706.1781 [hep-ph]].

[75] N. Ippolito, M. Ruggieri, D. Rischke, A. Sedrakian and F. Weber, Phys. Rev. D 77, 023004
(2008) arXiv:0710.3874 [astro-ph]].

[76] A. Sedrakian, Astron. \& Astrophys. 555, L 10 (2013) arXiv:1303.5380 [astro-ph.HE]].

[77] F. Sandin and D. Blaschke, Phys. Rev. D 75, 125013 (2007).

[78] M. Baldo, M. Buballa, F. Burgio, F. Neumann, M. Oertel and H. J. Schulze, Phys. Lett. B 562, 153 (2003).

[79] H. Grigorian, D. Blaschke and D. N. Aguilera, Phys. Rev. C 69, 065802 (2004).

[80] I. Shovkovy, M. Hanauske and M. Huang, Phys. Rev. D 67, 103004 (2003).

[81] M. Alford, M. Braby, M. W. Paris and S. Reddy, Astrophys. J. 629, 969 (2005).

[82] Note, that the strange current quark mass had to be corrected because of a mistake in the kaon mass formula employed in [36]. The corrected parameterization scheme has been implemented in an online tool developed by F. Sandin and is available under http://3fcs.pendicular.net/psolver 